2 J'en veux pour preuve l'invitation qu'il m'a faite et que j'ai dû décliner ( faute de temps...).

3 Grand dictionnaire terminologique: relatif aux caractéristiques propres à chaque individu, qui le distinguent des autres et qui déterminent sa façon particulière de réagir à son milieu et aux agents extérieurs.

4 Comme le futur CHUM si un jour on en voit la réalisation...

5 Klein semble confondre scène et décor.

6 Contrairement à celui qui voulait que la terre soit au centre de l'univers.

\section{Claude Courlet, L'économie territoriale, Grenoble, PUG, 2008, 135 p.}

Familier à de nombreux lecteurs d'O\&T, l'ami Courlet, professeur d'économie à l'Université Pierre-Mandès-France de Grenoble, aime bien faire concis. Sa décision de prendre le temps à nouveau de faire court (merci Voltaire) sera appréciée par le lecteur désireux de connaître l'essentiel sur des concepts abondamment utilisés dans la littérature actuelle en sciences régionales dites aussi territoriales comme le veut l'ouvrage qui précède cette recension. En reprenant des concepts maintes fois traités dans ces pages, Claude Courlet insiste sur le fait que de nouvelles exigences concurrentielles s'appuient de nos jours davantage sur des effets relationnels (les fameux réseaux) que sur les traditionnels effets de productivité. Ainsi, il montre que le territoire doit être abordé comme un système social doté de ressources relationnelles et non uniquement comme une simple entité spatiale pourvoyeuse de ressources physiques. Mais, pour s'assurer de bien se faire comprendre, l'auteur offre sa définition d'un territoire. Il y voit à la fois :

Un ensemble de facteurs aussi bien matériels qu'immatériels que la théorie économique a de longue date associé aux avantages que présentent la diminution des coûts de transaction

et l'existence d'économies externes;

Un système de relations économiques et sociales qui conduit au concept de capital social immortalisé par Putman;

- Un système de gouvernance local (au masculin dans texte) ${ }^{1}$.

Le territoire, précise bien Courlet, ne peut se réduire à l'interaction entre les trois dimensions que constituent les économies d'échelle, les coûts de transport et les économies d'agglomération. Au lieu de se voir considéré comme un ensemble de zones, il importe de percevoir le territoire comme un tissu (plus ou moins tricoté serré, comme on dit au Québec). Ce dernier pouvant se distinguer de tout autre par des caractéristiques qui lui sont propres, on doit en conclure que son développement exige des stratégies qui lui sont adaptées.

Dans un chapitre sur l'évolution du corpus théorique entourant la notion de territoire, une section intitulée Les nouvelles orientations de la théorie de la localisation, on peut lire que les économistes redécouvrent la géographie à travers la nouvelle géographie économique que l'on doit à P. Krugman ${ }^{2}$. Le grand public connaît davantage ce dernier pour ses chroniques dans le New York Times où il s'en est toujours pris avec virulence contre l'ancien locataire de la Maison Blanche que personne ne va regretter. L'auteur souligne que l'un des principaux mérites de Krugman fut de faire le lien entre les économies externes et les agglomérations industrielles régionales d'une part, et le commerce d'autre part. Ce qui aurait pour effet de remettre en question la thèse de la spécialisation flexible qui fait du commerce une variable dépendante du développement industriel. Mais, puisqu'il est question de spécialisation flexible, dans ce même chapitre, il fallait s'y attendre, l'auteur offre une synthèse concise de ce que sont les districts industriels en citant à son tour 
l'incontournable Beccatini ${ }^{3}$ se sentant obligé de rendre à César ce qu'il lui revient. Un encadré d'une page est consacré au plus célèbre des districts industriels celui de Prato à $15 \mathrm{Km}$ de Florence composé de 8500 entreprises $\mathrm{du}$ textile au sein desquelles 44000 personnes y trouvaient emplois récemment. J'utilise le passé car Courlet semble ignorer que Prato crie au secours. Au moment d'écrire ces lignes, pas moins de 4000 petites entreprises clandestines y embauchent des travailleurs chinois non moins clandestins, encouragés par leur gouvernement à quitter une Chine, elle aussi grande victime de la crise, à aller se faire voir ailleurs. Sans aide gouvernementale, Prato, tel que l'on connaît, sera rapidement chose du passé. Voilà la forme que prend le «péril jaune» de nos jours en Italie comme ailleurs ${ }^{4}$.

Dans un ouvrage où l'on traite de district industriel, on ne peut faire autrement que de s'intéresser également à leur extension: les systèmes de production locaux (ou localisés). Courlet en fait une présentation graphique fort simple qui désigne curieusement comme étant le triangle magique dont les trois sommets sont: agglomération, spécialisation et spécification (complémentarité, spécificités, coopération). C'est l'occasion choisie pour nous rappeler les fameux concepts de proximité organisée et géographique mis de l'avant par le duo Rallet-Torre. Et, juste avant de présenter Grenoble comme milieu innovateur, Courlet évoque ce qui est devenu aux yeux de plusieurs de nos amis de l'ASRDLF comme une vérité de l'Évangile (ne pas confondre avec une vérité de la Palice) : l'entreprise innovatrice est forgée par le milieu.

Dans un chapitre intitulé Les fondements territoriaux de la performance économique on trouve une citation d'un certain Barel pour qui l'homme est un animal territorialisateur (c'est mieux que terrifiant...). En conséquence, le territoire devient un « producteur » de mémoire locale ${ }^{5}$ et en même temps comme « créateur » d'un « code génétique » local (...) dont la valorisation permet de donner du sens aux actions et aux projets actuels et futurs. Le chapitre suivant intitulé Territoire, gouvernance locale et action publique débute, il va sans dire, par des définitions. Passons de suite à la gouvernance territoriale que l'auteur, avec raison, ne juge nécessaire de définir tout en précisant qu'elle ne se décrète pas (on s'en doutait) et qu'elle se veut un construit dans lequel les institutions sont largement imbriquées en y jouant un rôle d'intermédiation. C'est également dans ce chapitre que l'on trouve une section sur ce qui est en voie de devenir la tarte à la crème de nombreux collègue hexagonaux comme en font foi plusieurs recensions de cette chronique: les pôles de compétitivité. Ne se limitant pas à tout simplement les définir, Courlet innove en distinguant deux catégories de pôles de compétitivité : une première dite à dimension internationale composée de grands groupes industriels et une seconde caractérisée par des activités traditionnelles comme le veut le sempiternel exemple du secteur du décolletage (sic) de la vallée de l'Arve (les Québécois de ma génération, avec le cardinal Léger, ont connu, eux, la vallée de larmes...).

Un dernier et très bref chapitre, Perspectives pour les régions pauvres n'a rien à voir avec les nôtres puisqu'il s'agit en fait d'exemples de pays du tiers-monde, ou en émergence (comme le Brésil) autrefois désignés comme étant en voie de développement ou encore plus justement: sous-développés ou moins développés suivant que l'on respecte ou pas la rectitude politique qui interdit d'appeler un chat un chat.

On aura compris que ce modeste ouvrage constitue un utile vade mecum de tout de qui entoure la question territoriale. 
1 Je préfère voir le mot local accordé avec le mot gouvernance même si ici le système est manifestement localisé.

2 Auréolé en 2008 du prix de la Banque de Suède en l'honneur de Gustav Nobel. Ce prix est faussement désigné comme étant le prix Nobel de l'économie. Mais : Who cares?

3 Le même Beccatini, que j'ai rencontré à Genève en 1990, ne s'attendait sûrement pas à se voir cité autant.

4 À Alger on commence à voir des vendeurs de rue chinois... On sait que le gouvernement chinois offre des subventions aux agences de voyages qui offrent un billet aller seulement aux chômeurs à qui on délivre sans réserve le passeport nécessaire.

5 Parlons-en à Fred Pellerin...

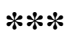

Michel Arcand, La gestion du changement : vingt-et-une questions pour mieux appréhender et gérer les transformations organisationnelles, Montréal, Éditions Nouvelles, 2007, 195 p.

Une fois n'est pas coutume, avec cet ouvrage qui a remporté le Prix du livre d'affaires 2008, il n'est pas question de territoires mais uniquement d'organisations. De toute façon, comme il n'y a pas de territoires sans organisations, le lecteur ne me tiendra pas rigueur pour le choix d'un ouvrage dont l'auteur est un de mes anciens étudiants. Ce dernier, en plus d'avoir remporté ce prix, s'est vu, pour la même année, octroyer le prix du meilleur enseignant de l'UQTR où il est professeur en développement organisationnel depuis 2001. Si Michel Arcand n'a pas tardé à laisser sa marque dans la ville de Laviolette, l'ouvrage qu'il nous présente ici, pour l'essentiel, est issu d'une expérience pédagogique vécue au niveau du MBA à la Haute école de gestion de Fribourg où il intervient régulièrement. En publiant ce livre, inconsciemment, l'auteur fait un lien avec la dynamique territoriale (ou c'est à ses lecteurs de le faire) lorsqu'il écrit que plus que jamais dans l'histoire économique moderne, il n'a été aussi important de " mailler » aspects humains et activités d'affaires. En se situant à l'échelon de l'entreprise, M. Arcand soutient que le meilleur des projets n'aura que très peu d'écho sans l'adhésion pleine et entière des employés. À nous de dégager qu'il en est de même pour tout projet à l'intérieur d'un territoire donné. Les exemples ne manquent pas au Québec. Il faut donc qu'il $y$ ait connivence entre l'organisation et l'ensemble des acteurs concernés.

Dans la préface, Jacques Perani, de l'Université Robert Schuman de Strasbourg, cite Edgar Faure, homme politique français et académicien, décédé il y a une vingtaine d'années, qui, à l'occasion d'une xième tentative de réforme de l'Éducation nationale ${ }^{1}$ a déclaré : "En décrétant le changement, l'immobilisme s'est mis en marche et je ne sais plus comment l'arrêter.» Cet immobilisme, les Montréalais en savent quelque chose où les groupes de pression des différents arrondissement font la pluie et le beau temps ${ }^{2}$. Ainsi $O \& T$ pourra célébrer son $25^{\mathrm{e}}$ anniversaire de création alors que les Montréalais seront toujours en attente de leur CHUM. L'auteur, quant à lui, offre comme histoires d'horreur le stade olympique, l'édifice de la Caisse de dépôt et de placement du Québec (les 40 milliards n'étaient pas encore volatilisés au moment d'écrire le volume) et le métro de Laval.

Mais, transportons-nous à Fribourg où débute un cours sur la gestion stratégique du changement, avec la première des 21 questions : " Pourquoi devons-nous continuellement changer? » Mais, de quoi parle-t-on? M. Arcand juge la définition suivante trop académique: "La gestion du changement relève d'une activité de gestion à la fois stratégique et opérationnelle initiée par la direction de l'entreprise dont le but premier est de permettre aux différents acteurs de 